Department of Mathematics

\title{
Optimal scheduling for replacing perimeter guarding unmanned aerial vehicles
}

Oleg Burdakov, Patrick Doherty and Jonas Kvarnström

LiTH-MAT-R--2014/09--SE 
Department of Mathematics

Linköping University

S-581 83 Linköping 


\title{
Optimal scheduling for replacing perimeter guarding unmanned aerial vehicles
}

\author{
Oleg Burdakov ${ }^{\mathrm{a}, 1}$, Patrick Doherty ${ }^{\mathrm{b}}$ and Jonas Kvarnström ${ }^{\mathrm{b}}$ \\ a Department of Mathematics, Linköping University, SE-581 83 Linköping, Sweden \\ b Department of Computer and Information Science, Linköping University, Sweden
}

\begin{abstract}
Guarding the perimeter of an area in order to detect potential intruders is an important task in a variety of security-related applications. This task can in many circumstances be performed by a set of camera-equipped unmanned aerial vehicles (UAVs). Such UAVs will occasionally require refueling or recharging, in which case they must temporarily be replaced by other UAVs in order to maintain complete surveillance of the perimeter. In this paper we consider the problem of scheduling such replacements. We present optimal replacement strategies and justify their optimality.
\end{abstract}

Keywords: scheduling problem; optimal replacement strategies; perimeter guarding; unmanned aerial vehicles.

\section{Introduction}

To determine how a team of autonomous robots should guard the perimeter of a large area against a potential intruder, we need to answer two questions: How do we place the robots, and when do we replace them?

The question of placement has already been extensively covered in the literature. For example, static placement problems concern determining how to place sensors (not necessarily associated with robots) in fixed locations. This includes the well-known art gallery problem [16] as well as many coverage problems $[5,6]$. In cases such as underwater surveillance, sensors can have properties such that no placement can guarantee detection, but it can still be possible to find a placement of sensors around a perimeter that maximizes the detection probability [15]. In contrast, dynamic placement problems concern determining suitable movement strategies for robots, which is particularly useful when the number of robots is insufficient for completely covering the desired area given static locations $[2,3,1]$. When the target attempts to avoid detection, this turns into a pursuit/evasion problem $[2,12,17]$. In this case, the algorithms may also cover the task of tracking an intruder once it has been detected.

In this paper we focus on the second question, that of replacing UAVs. We consider a general case where $n$ guarding UAVs are spread out along a perimeter to be continuously guarded against intruders. The UAVs may be statically placed in locations covering the entire perimeter, or they may move in deterministic or unpredictable patterns in order

\footnotetext{
${ }^{1}$ Corresponding author. Tel.: +46 13 281473. E-mail address: oleg.burdakov@liu.se
} 
to maximize the probability of intruder detection, possibly using one of the methods referenced above. In either case, their individual endurance will not be sufficient for an extended mission. Finding a strategy to replace such UAVs while maintaining sufficient coverage is essential in surveillance applications, and is equally relevant regardless of whether the placement of UAVs is static or dynamic.

In some scenarios, additional UAVs could be available and could be on stand-by to replace UAVs whose battery or fuel levels are low. A UAV that needs to recharge or refuel would then be replaced immediately by a stand-by UAV, and when it returns from the base, it would be placed on standby for future replacements. Alternatively, all available UAVs can actively participate in the surveillance mission to improve coverage. Then any UAV whose battery level is low can temporarily return to base, leaving the remaining $n-1$ UAVs to continue guarding the perimeter until it returns. Below, both cases will be considered replacement: A UAV leaves, and is immediately or eventually replaced with another or with a recharged or refueled version of itself. Thus, UAVs on stand-by will also be considered guarding UAVs.

Note also that refueling is equivalent to recharging or automatically replacing batteries [18] except for the difference in time requirements. For brevity, we will therefore limit the discussion below to the use of batteries that are charged and discharged, without loss of generality. The guarding UAVs are assumed to be fully charged at the initial time, $t=0$. Similarly, each replacement UAV is assumed to be fully charged at the time it returns to the perimeter guarding mission.

When an intruder is detected, one of the two UAVs closest to the intruder should become a tracking $U A V$, leaving the perimeter in order to track the intruder. The assumption is then that the remaining UAVs are sufficient to continue guarding the perimeter, either with complete coverage or with a sufficient probability of detection. We then want to schedule replacements in a way that maximizes the worst case battery charge of the tracking UAV, while ensuring that the guarding UAVs remain able to return to the base to recharge.

Some approaches to recharging in the literature do not consider scheduling, instead assuming each robot will use a dedicated recharging station whenever necessary [8]. Others do consider scheduling but focus on objectives and requirements that differ from the ones considered here. In some cases UAVs must continuously follow known trajectories through space without interruption [11], which necessitates the scheduling of a replacement that takes over at the precise point where the original UAV leaves the mission. In other cases, there is a mobile robot with the ability to recharge others, leading to the problem of scheduling meetings between robots as well as computing paths leading to the selected meeting points $[14,10,4,13]$. These solutions are not suitable for the case we consider here, where the recharging station is immobile and where no individual UAV is indispensable for guarding the perimeter. There is also work focusing on scenarios where robots can temporarily leave a mission for refueling at any time, without the need to keep a certain number of robots active, but where multiple robots share refueling stations and must schedule their activities accordingly [9]. This work is also not applicable to the situation considered here.

This paper presents an alternative solution adapted to the conditions specific to the type of perimeter guarding mission discussed above. In such missions, even if the area 


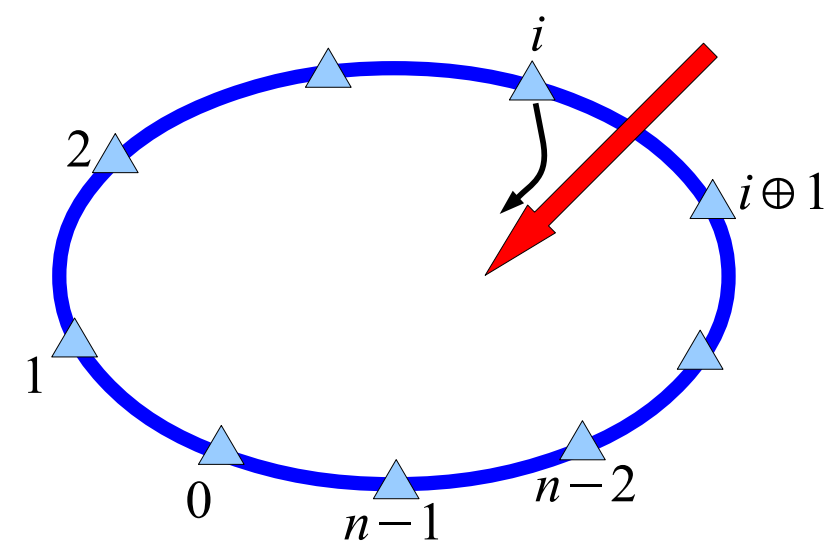

Figure 1: Perimeter guarding UAVs; an intrusion of the perimeter segment between UAVs $i$ and its adjacent $\mathrm{UAV} i \oplus 1$

guarded by each UAV may change dynamically, there is no need for guarding UAVs to change order along the perimeter. This facilitates the construction of a replacement strategy where guarding UAVs are replaced one by one every fixed time interval $\tau$, a strategy that can be proven optimal under the given requirements.

As already noted, when a perimeter intrusion is identified in the area between a pair of adjacent UAVs, like in Fig. 1, the one with the higher battery charge, say UAV $i$, will attempt to follow and track the intruder.

Any such pair of adjacent UAVs is characterized by the higher battery charge of the two UAVs. This is called the pair's tracking charge. The lowest tracking charge over all adjacent pairs and all time is called the critical tracking charge, and the corresponding pair of adjacent UAVs is called the weakest. Another important characteristic of the replacement strategy to be developed is the minimal charge over all guarding UAVs, which is called the critical guarding charge. The replacement strategy must be constructed so that this charge is always sufficient to allow the corresponding UAV to come back to the base for recharging.

Since it is not a priori known which pair of UAVs may be affected by an intrusion, it is natural to require from the replacement strategy that it maximizes the critical tracking charge and keeps the critical guarding charge at the admissible level at all time. This is the main problem that we address in this paper.

\section{$1.1 \quad$ Organization}

The paper is organized as follows. In Section 2, the scheduling problem is formulated. Periodic replacement strategies play an important role in our development of optimal strategies. Their useful properties are considered in Section 3. In Section 4, we present optimal replacement strategies, justify their optimality and discuss their uniqueness. In Section 5, we draw conclusions and discuss future work. 


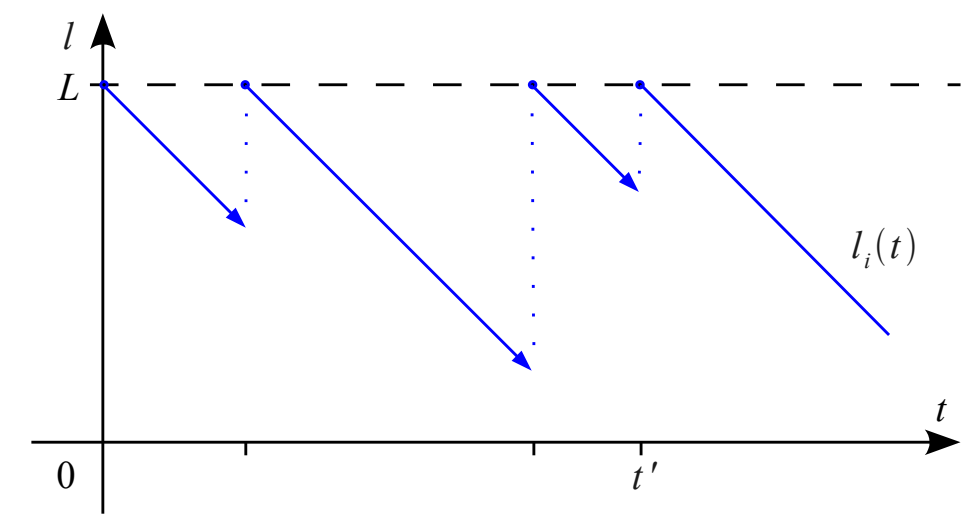

Figure 2: Charge of UAV $i$ vs. time

\section{Problem formulation}

The guarding UAVs are assumed to be numbered as shown in Fig. 1, where the notation

$$
i \oplus 1= \begin{cases}i+1, & \text { if } i \neq n-1 \\ 0, & \text { if } i=n-1\end{cases}
$$

comes from the modular arithmetic [7]. In general, given integers $i$ and $j, i \oplus j$ will stand for the addition modulo $n$, which is equal to the quotient remainder in $(i+j) / n$. We similarly denote $i \ominus j=i \oplus(-j)$. For simplicity, $(\bmod n)$ will be omitted in the modular equivalence $i \equiv j(\bmod n)$. Let $N=\{0,1,2, \ldots, n-1\}$ stand for the set of all guarding UAVs. We refer to the pair of adjacent $\operatorname{UAVs}(i, i \oplus 1)$ as pair $i$.

The battery charge $l_{i}(t)$ of UAV $i$ is assumed to decrease linearly with time as follows

$$
l_{i}(t)=L-c\left(t-t^{\prime}\right),
$$

where the positive scalars $L$ and $c$ denote the full battery charge and discharge rate, respectively, and $t^{\prime}$ stands for the latest time, before $t$, when UAV $i$ was replaced. An example of such a function is presented by Fig. 2 .

The tracking charge introduced in the previous section is computed for pair $i$ by the formula

$$
\bar{l}_{i}(t)=\max \left\{l_{i}(t), l_{i \oplus 1}(t)\right\} .
$$

This function is determined by a chosen replacement strategy. It has a form similar to $l_{i}(t)$ :

$$
\bar{l}_{i}(t)=L-c\left(t-t^{\prime}\right)
$$

with the difference that $t^{\prime}$ here stands for the latest time before $t$ when any UAV in pair $i$ was replaced.

Any replacement strategy $s$ can be presented as a sequence of integer numbers $\nu_{0}, \nu_{1}, \nu_{2}, \ldots$ from the set $N$. Each number $\nu_{k}$ indicates that UAV $\nu_{k}$ should be replaced at time

$$
t_{k}=k \tau, \quad k=0,1,2, \ldots
$$


The set of all replacement strategies is denoted by $S$. This is actually the set of all infinite sequences of numbers from $N$.

The critical tracking charge introduced in the previous section is defined as

$$
\bar{l}(s)=\min _{i \in N} \inf _{t \geq 0} \bar{l}_{i}(t) .
$$

The critical guarding charge is computed by the formula

$$
\underline{l}(s)=\min _{i \in N} \inf _{t \geq 0} l_{i}(t) .
$$

We obviously have $\underline{l}(s) \leq \bar{l}(s)$ because $l_{i}(t) \leq \bar{l}_{i}(t)$ for all $t \geq 0$ and $i \in N$.

As was mentioned in Section 1, the critical guarding charge should be above a given charge level, denoted here by $l_{\text {min }}$, which is sufficient to allow any guarding UAV to come back to the base for recharging. The replacement scheduling problem outlined in Section 1 can now be formulated as follows:

$$
\max _{s \in S}\left\{\bar{l}(s): \underline{l}(s) \geq l_{\min }\right\} .
$$

Since, at any interval $\left[t_{k}, t_{k+1}\right)$, the function $\min \left\{\bar{l}_{i}(t): i \in N\right\}$ decreases linearly with $t$, formula (3) can be written as

$$
\bar{l}(s)=\Lambda(s)-c \tau,
$$

where

$$
\Lambda(s)=\min _{k \geq 1, i \in N} \bar{l}_{i}\left(t_{k}\right)
$$

Similarly, we can rewrite formula (4) as

$$
\underline{l}(s)=\lambda(s)-c \tau,
$$

where

$$
\lambda(s)=\min _{k \geq 1, i \in N} l_{i}\left(t_{k}\right) .
$$

Relations (6) and (7) allow us to present problem (5) in the following equivalent form:

$$
\max _{s \in S}\left\{\Lambda(s): \lambda(s) \geq l_{\min }+c \tau\right\} .
$$

Note that only the discrete time values $l_{i}\left(t_{k}\right)$ are involved in this problem formulation. We use this property in the next two sections for developing replacement strategies and justifying their optimality.

\section{$3 \quad$ Periodic strategies}

We call strategy $s=\left\{\nu_{0}, \nu_{1}, \nu_{2}, \ldots\right\} \in S$ periodic if the segment $\left\{\nu_{0}, \nu_{1}, \ldots, \nu_{n-1}\right\}$ of this sequence is a permutation of the sequence $\{0,1, \ldots, n-1\}$ and

$$
\nu_{k+n}=\nu_{k}, \quad \forall k \geq 0 .
$$


Our analysis of such strategies will be based on the fact that $l_{i}(t)$ and $\bar{l}_{i}(t)$ are periodic functions with the period $T=n \tau$.

Periodic strategies play an important role in our development of optimal strategies. One of their key properties is that $\underline{l}(s)$ attains its maximal value all over $s \in S$ if and only if $s$ is periodic. It is an implication of the following result.

Lemma 1 If $s \in S$ is a periodic strategy, then $\underline{l}(s)=L-c T$. If $s \in S$ is not periodic, then $\underline{l}(s)<L-c T$.

Proof. The equality $\underline{l}(s)=L-c T$ immediately follows from the fact that, for any periodic strategy $s$, each guarding UAV is replaced once every fixed time interval $T$.

Consider any $s \in S$ which is not periodic. This means that, for this strategy, there exists $k \geq 1$ such that $\nu_{k} \neq \nu_{k+n}$. Suppose, on the contrary, that

$$
\underline{l}(s) \geq L-c T \text {. }
$$

To meet this requirement, every number $i \in N$ should appear at least twice in the sequence of $2 n$ numbers $\nu_{k}, \nu_{k+1}, \ldots, \nu_{k+2 n-1}$. Moreover, it should appear exactly twice because $N$ is composed of $n$ numbers. Inequality (9) implies that there should exist $m \leq n$ such that $\nu_{k}=\nu_{k+m}$. Since $\nu_{k} \neq \nu_{k+n}$, we have $m<n$. Therefore, the number $\nu_{k}$ appears twice in the first half of the mentioned sequence of $2 n$ numbers. Then it does not appear in the second half of the sequence and, for this reason,

$$
\underline{l}(s) \leq l_{\nu_{k}}((k+2 n-1) \tau)<L-c T .
$$

This contradicts assumption (9) and accomplishes the proof of lemma.

This result allows us to draw the following practical conclusion about the admissible time interval between two sequential UAV replacements. Any periodic strategy $s$ is feasible in problem (5) if and only if

$$
\tau \leq \frac{L-l_{\min }}{c n}
$$

Note that in practice, $\tau$ is also bounded from below by the time it takes for each UAV to return to the base station and recharge (or refuel or change batteries). In the case where there exist $m>0$ additional "standby" UAVs that are not part of the actual surveillance, this lower bound can be decreased by a factor of $m+1$ assuming that multiple UAVs can be recharged in parallel. For example, with 2 standby UAVs, the effective recharge time is reduced to a third. The maximal achievable critical tracking charge will of course depend on which value of $\tau$ is chosen (the more often UAVs are replaced, the less energy will have been consumed between two replacements of the same UAV, and the greater the critical tracking charge). However, the optimal replacement order is not affected by the value of $\tau$ as long as this value remains within the given bounds.

From now on, we assume that $S$ is the set of all periodic strategies. In our analysis of periodic strategies, we shall appeal to a directed cycle graph $G=(K, E)$, where $K=\{0,1,2, \ldots, n-1\}$ and $E=\{(k, k \oplus 1): k \in K\}$ are its sets of nodes and edges, 
respectively. Given $k^{\prime}, k^{\prime \prime} \in K$, let $d\left(k^{\prime}, k^{\prime \prime}\right)$ denote the distance defined as the number of edges in the directed path from $k^{\prime}$ to $k^{\prime \prime}$. Obviously,

$$
d\left(k^{\prime}, k^{\prime \prime}\right)=k^{\prime \prime} \ominus k^{\prime}
$$

Note that any periodic strategy $s \in S$ can be viewed as a bijection $\kappa_{s}: N \rightarrow K$ such that $\nu_{\kappa_{s}(i)}=i$, for all $i \in N$.

Given a bijection $\kappa_{s}$, if UAV $i$ is replaced at a moment $\mathrm{t}$, then the first of the subsequent replacements of $\mathrm{UAV} i \oplus 1$ occurs at the moment

$$
t+\tau d\left(\kappa_{s}(i), \kappa_{s}(i \oplus 1)\right)
$$

Similarly, for the length of the time interval between the replacement of UAV $i \oplus 1$ and the first of the subsequent replacements of $\mathrm{UAV} i$, we have

$$
\tau d\left(\kappa_{s}(i \oplus 1), \kappa_{s}(i)\right)
$$

Combining this with (11), we obtain

$$
\inf _{t \geq 0} \bar{l}_{i}(t)=L-\tau D_{i}(s),
$$

where

$$
D_{i}(s)=\max \left\{d\left(\kappa_{s}(i), \kappa_{s}(i \oplus 1)\right), d\left(\kappa_{s}(i \oplus 1), \kappa_{s}(i)\right)\right\} .
$$

Since the length of any nontrivial cycle in our graph $G$ is equal to n, we have

$$
d\left(\kappa_{s}(i), \kappa_{s}(i \oplus 1)\right)+d\left(\kappa_{s}(i \oplus 1), \kappa_{s}(i)\right)=n, \quad \forall i \in K .
$$

This and the definition of distance (11) allow us to rewrite the formula for $D_{i}(s)$ as

$$
\begin{aligned}
D_{i}(s) & =\max \left\{d\left(\kappa_{s}(i), \kappa_{s}(i \oplus 1)\right), n-d\left(\kappa_{s}(i), \kappa_{s}(i \oplus 1)\right)\right\} \\
& =\max \left\{\kappa_{s}(i \oplus 1) \ominus \kappa_{s}(i), n-\left[\kappa_{s}(i \oplus 1) \ominus \kappa_{s}(i)\right]\right\}
\end{aligned}
$$

Observe that

$$
\Lambda(s)=L-c \tau \max _{i \in N}\left(D_{i}(s)-1\right) .
$$

Then equation (6) can be written as

$$
\bar{l}(s)=L-c \tau \max _{i \in N} D_{i}(s) .
$$

By this means our replacement scheduling problems (5), in case of periodic strategies, is reduced to the problem

$$
\min _{s \in S} \max _{i \in N} D_{i}(s)
$$

Here it is assumed that $\tau$ is chosen in accordance with (10).

Note that our optimal periodic strategies introduced in the next section admit an easy derivation of $D_{i}(s)$ and then straightforward calculation of $\bar{l}(s)$ by formula (15). 


\section{Optimal replacement schedule}

Before introducing our optimal strategies, we will find an upper bound for any $\bar{l}(s)$ including, of course, the optimal objective function value in problem (5). The upper bound cannot be improved by any periodic strategy which is infeasible in problem (5). This result is formulated as follows.

Lemma 2 For any $s \in S$, if $n$ is odd then

$$
\bar{l}(s) \leq \bar{l}_{\text {odd }}
$$

else

$$
\bar{l}(s) \leq \bar{l}_{\text {even }},
$$

where

$$
\bar{l}_{\text {odd }}=L-\frac{c(n+1) \tau}{2} \text { and } \quad \bar{l}_{\text {even }}=L-\frac{c(n+2) \tau}{2} .
$$

Proof. For the odd values of $n$, equation (14) yields the inequality

$$
D_{i}(s) \geq \frac{n+1}{2}
$$

which holds for any $s \in S$. This inequality, along with (15), proves (16).

Consider the case of even values of $n$. Let $s$ be any periodic strategy. Consider any $i \in N$. If $\kappa_{s}(i \oplus 1) \neq \kappa_{s}(i) \oplus n / 2$, then (14) gives the inequality

$$
D_{i}(s) \geq \frac{n+2}{2}
$$

which, by (15), implies (17). Suppose now that $\kappa_{s}(i \oplus 1)=\kappa_{s}(i) \oplus n / 2$. This excludes the case when $\kappa_{s}(i \oplus 2)=\kappa_{s}(i \oplus 1) \oplus n / 2$ because $\kappa_{s}(i \oplus 1) \oplus n / 2=\kappa_{s}(i)$. Therefore, the inequality

$$
D_{i \oplus 1}(s) \geq \frac{n+2}{2}
$$

holds and, by (15), this finally proves (17).

The proof of Lemma 2 is not constructive because it exploits some general properties of periodic strategies without suggesting any specific strategy. We are now going to present some strategies which make tight the bounds (16) and (17).

For the odd values of $n$, we suggest a simple strategy denoted by $s_{o d d}$ and defined by the recursive formula:

$$
\begin{aligned}
& \nu_{0}=0, \\
& \nu_{k}=\nu_{k-1} \oplus 2, \quad k=1,2,3, \ldots .
\end{aligned}
$$

If, for example, $n=7$, it produces the UAV replacement sequence

$$
\{0,2,4,6,1,3,5,0,2,4,6,1,3, \ldots\}
$$

(see Fig. 3). The following result summarizes the main properties of strategy (18). 


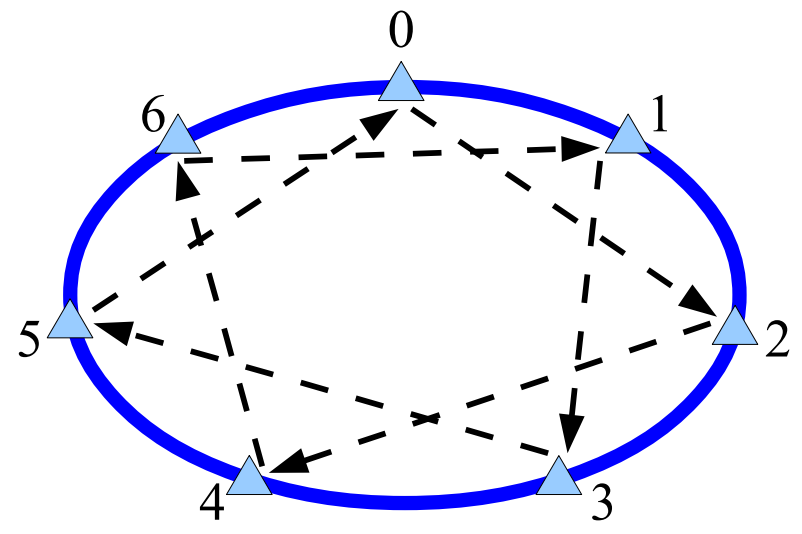

Figure 3: Optimal replacement sequence produced by $s_{\text {odd }}$ for 7 UAVs

Theorem 3 Let $n$ be an odd number. Suppose that $\tau$ satisfies inequality (10). Then strategy (18) is periodic. Moreover, it is an optimal solution of problem (5), and the optimal value of the objective function in this problem is $\bar{l}_{\text {odd }}$.

Proof. It can be easily verified that strategy (18) is periodic. Then due to assumption (10) and by Lemma 1, this strategy is feasible in problem (5).

Consider any $i \in N$. The recursive formula (18) gives $i \equiv 2 \kappa_{s_{\text {odd }}}(i)$. For odd values of $n$, we have $2^{-1} \equiv(n+1) / 2$ (see, e.g., [7]). Combining these two relations, we obtain

$$
\kappa_{s_{\text {odd }}}(i) \equiv \frac{n+1}{2} \cdot i \quad \text { and } \quad \kappa_{s_{\text {odd }}}(i \oplus 1) \equiv \kappa_{s_{\text {odd }}}(i) \oplus \frac{n+1}{2} \text {. }
$$

Consequently,

$$
\kappa_{s_{\text {odd }}}(i \oplus 1) \ominus \kappa_{s_{\text {odd }}}(i)=\frac{n+1}{2} .
$$

Then, by formula (14), we get

$$
D_{i}\left(s_{\text {odd }}\right)=\max \left\{\frac{n+1}{2}, \frac{n-1}{2}\right\} .
$$

By substituting this equation into (15), we conclude that $\bar{l}\left(s_{\text {odd }}\right)$ equals the upper bound in (16). Then Lemma 2 finally implies that the strategy $s_{\text {odd }}$ solves problem (5).

For the even values of $n$, we suggest a more intricate and far less obvious strategy than $s_{\text {odd }}$. It is denoted by $s_{\text {even }}$ and defined recursively for $k=0,1,2, \ldots, n-1$ as follows:

$$
\begin{array}{lll}
\nu_{0}=0, & \nu_{k}=\nu_{k-1} \ominus(-1)^{k} 2 k, & k=1, \ldots, n / 2-1, \\
\nu_{n / 2}=1, & \nu_{k}=\nu_{k-1} \oplus(-1)^{k-n / 2} 2(k-n / 2), & k=n / 2+1, \ldots, n-1 .
\end{array}
$$

This sequence is obviously a permutation of $\{0,1, \ldots, n-1\}$. The whole sequence $\nu_{k}$ is obtained by periodically extending sequence (20). If, for example, $n=8$, it produces the replacement sequence

$$
\{0,2,6,4,1,7,3,5,0,2,6,4,1,7,3,5, \ldots\}
$$




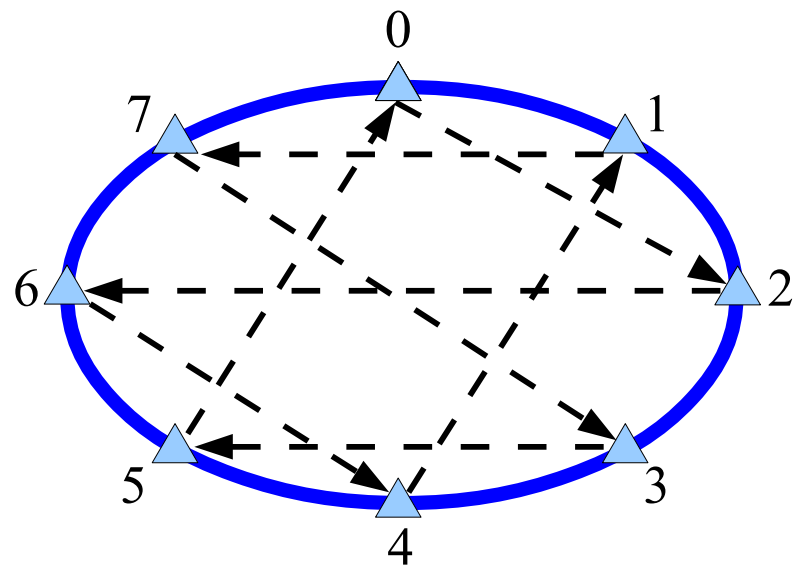

Figure 4: Optimal replacement sequence produced by $s_{\text {even }}$ for 8 UAVs

(see Fig. 4). The following result summarizes the main properties of the presented strategy.

Theorem 4 Let $n$ be an even number. Suppose that $\tau$ satisfies inequality (10). Then strategy $s_{\text {even }}$ is an optimal solution of problem (5), and the optimal value of the objective function in this problem is $\bar{l}_{\text {even }}$.

Proof. Based on the recursion (20), one can easily derive the relations

$$
\kappa_{\text {seven }}(i)= \begin{cases}0, & \text { if } i=0, \\ i-1, & \text { if } i \text { is even and } 2 \leq i \leq n / 2, \\ n-i, & \text { if } i \text { is even and } n / 2<i \leq n-2, \\ n / 2+i-1, & \text { if } i \text { is odd and } 1 \leq i \leq n / 2, \\ 3 n / 2-i, & \text { if } i \text { is odd and } n / 2<i \leq n-1\end{cases}
$$

and

$$
\kappa_{\text {seven }}(i \oplus 1)= \begin{cases}n / 2+i, & \text { if } i \text { is even and } 0 \leq i<n / 2, \\ 3 n / 2-i-1, & \text { if } i \text { is even and } n / 2 \leq i \leq n-2 \\ i, & \text { if } i \text { is odd and } 1 \leq i<n / 2, \\ n-i-1, & \text { if } i \text { is odd and } n / 2 \leq i \leq n-1\end{cases}
$$

They give

$$
D_{i}\left(s_{\text {even }}\right)= \begin{cases}n / 2, & \text { if } i=0 \text { or } i=n / 2 \\ n / 2+1, & \text { otherwise }\end{cases}
$$

After substituting this equation in (15), we observe that $\bar{l}\left(s_{\text {even }}\right)$ equals the upper bound in (17). Then, by Lemma 2, the strategy $s_{\text {even }}$ solves problem (5).

Note that the presented optimal strategies are not unique. Indeed, if the guarding UAVs are counted counterclockwise, the strategies formally defined by (18) and (20) are, obviously, also optimal. Furthermore, it can be easily verified that, if $\left\{\nu_{k}, \nu_{k+1}, \ldots\right\}$ is a truncated subsequence of either $s_{\text {odd }}$ or $s_{\text {even }}$, this subsequence is also an optimal strategy. 
This suggests that any optimal periodic strategy remains optimal under some invariant changes in circular numbering of UAVs, namely, when the circular direction changes between clockwise and counter-clockwise, and when the numbering is shifted clockwise or counter-clockwise.

These, and only these, invariant changes $P: N \rightarrow N$ have the property that $P$ is a bijection which preserves the set of neighbors in the sense of the following set equivalence

$$
\{P(i) \ominus 1, P(i) \oplus 1\}=\{P(i \ominus 1), P(i \oplus 1)\}, \quad \forall i \in N .
$$

The optimality for the changed numbering follows from the fact that any $P$ of this property does not change the set of distances between the neighbors involved in calculating the objective function value $\bar{l}(s)$ by formulas (12) and (15). This shows that any optimal strategy, not only (18) or (20), remains optimal under any change that possesses the property (21). Given a periodic sequence $s$ and a bijection $P$, let $P(s)$ stand for the sequence produced from the $s$ by applying the $P$ to each member of the $s$.

We now consider the uniqueness of the strategies $s_{\text {odd }}$ or $s_{\text {even }}$. The following result shows that the $s_{\text {odd }}$ is unique up to the mentioned invariant changes in circular numbering of UAVs.

Theorem 5 Let $n$ be an odd number. Suppose that $s \in S$ is an optimal solution of problem (5). Then there exists a bijection $P: N \rightarrow N$ which satisfies (21) and is such that $s=P\left(s_{\text {odd }}\right)$.

Proof. The invariance under any shifting of the numbering allows us to assume, without loss of generality, that the sequence produced by the $s$ begins with 0 , i.e. $\kappa_{s}(0)=0$.

The optimality of the $s$ dictates that

$$
D_{i}(s)=\frac{n+1}{2}, \quad \forall i \in N,
$$

otherwise $\bar{l}(s)$ would not attain $\bar{l}_{\text {odd }}$, the optimal objective function value. This means that, for each $i \in N$, there exist only two alternatives, namely,

$$
\text { either } \kappa_{s}(i \oplus 1)=i \oplus \frac{n+1}{2} \text {, or } \kappa_{s}(i \oplus 1)=i \oplus \frac{n-1}{2} \text {. }
$$

Assume that the $s$ is such that the first of the alternatives holds for $i=0$. In this case, (19) indicates the coincidence $\kappa_{s}(1)=\kappa_{s_{\text {odd }}}(1)$. For $i=1$, the second alternative in $(22)$ cannot hold because this would result in the equality $\kappa_{s}(2)=0=\kappa_{s}(2)$ which contradicts the assumption that $\kappa_{s}$ is a bijection. The first alternative, in the case of $i=1$, gives $\kappa_{s}(2)=1=\kappa_{\text {sodd }}(2)$. By following the same reasoning, sequentially for $i=2,3, \ldots, n-1$, it can be easily seen that the only true alternative in (22) is the first one, and also that this alternative results in $\kappa_{s}(i \oplus 1)=\kappa_{s_{\text {odd }}}(i \oplus 1)$. This proves that, under our assumption, $s=s_{\text {odd }}$ because the $\kappa$ is a bijection.

Assume now that the second alternative in (22) holds for $i=0$. Let $s_{\text {odd }}^{\prime}$ stand for the optimal strategy that produces the replacement sequence

$$
\begin{aligned}
& \nu_{0}=0, \\
& \nu_{k}=\nu_{k-1} \ominus 2, \quad k=1,2,3, \ldots .
\end{aligned}
$$




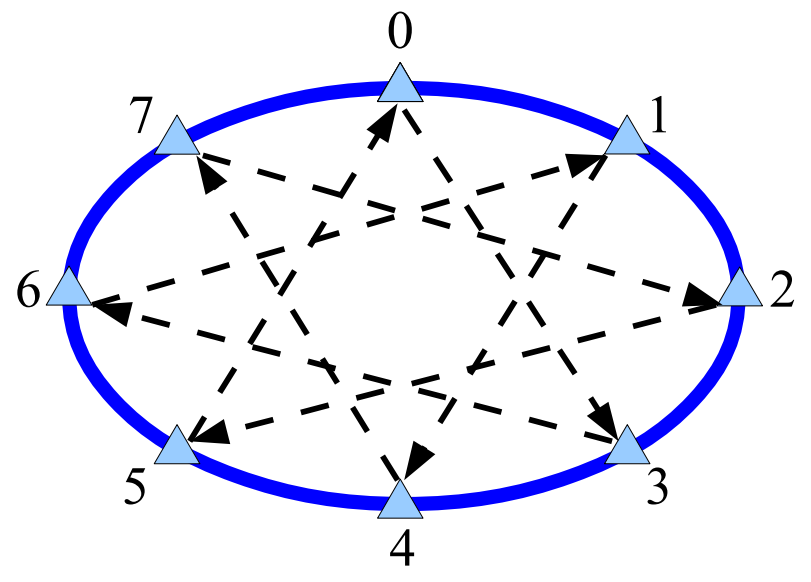

Figure 5: Optimal replacement sequence produced by $s_{\text {even }}^{\prime}$ for $8 \mathrm{UAVs}$

Clearly, $s_{\text {odd }}^{\prime}=P\left(s_{\text {odd }}\right)$, where the bijection $P$ is such that

$$
P(i) \equiv-i, \quad \forall i \in N
$$

It is also obvious that the $P$ satisfies $(21)$ as it corresponds to the counter-clockwise numbering of UAVs. Like above, it can be shown, sequentially for $i=1,2, \ldots, n-1$, that the strategy $s$ must satisfy the second alternative in $(22)$ and that it coincides with $s_{\text {odd }}^{\prime}$. This accomplishes our proof of the fact that the strategy $s_{\text {odd }}$ is unique up to the invariant changes that satisfy (21).

We will show that, in contrast to the $s_{\text {odd }}$, the strategy $s_{\text {even }}$ is not unique if to consider the uniqueness up to the aforementioned invariant changes. Indeed, consider a periodic strategy $s_{\text {even }}^{\prime}$ which produces for $n=8$ the replacement sequence

$$
\{0,3,6,1,4,7,2,5,0,3,6,1,4,7,2,5, \ldots\}
$$

(see Fig. 5). It can be easily seen that this sequence is optimal and also that there exists no bijection $P$ which satisfies $(21)$ and is such that $s_{\text {even }}^{\prime}=P\left(s_{\text {even }}\right)$.

\section{Conclusions and future work}

In this paper we considered the problem of scheduling replacements of UAVs in a perimeter guarding task. The main results are the following. A practical importance of periodic replacement strategies was justified. Based on this result, a minimal time interval between two sequential UAV replacements was derived. Replacement strategies were introduced separately for odd and even number of UAVs, and their optimality was proven. It was also proven that the replacement strategy $s_{\text {odd }}$ is unique up to the invariant transformations discussed at the end of the previous section.

We plan to consider a possibility of finding all replacement strategies which are optimal in the case of even number of UAVs. 
The problem formulation was based on the assumption that all UAVs have the same full battery charge $L$ and the same discharge rate $c$. However, the results in the article in fact only depend on all UAVs having the same endurance $L / c$. To handle multiple UAVs with different charge but identical endurance, set $L=1$ to represent a full battery charge and adapt $c$ accordingly. Small differences in endurance can also be handled using a distinct $c_{i}$ for each UAV and letting $c=\max _{i} c_{i}$. The resulting replacement strategies remain sound, though they do not necessarily maximize the critical tracking charge. In the future we intend to analyze the more difficult case of optimally replacing UAVs with widely varying endurance $L_{i} / c_{i}$.

\section{Acknowledgements}

This work is partially supported by the EU FP7 project SHERPA (grant agreement 600958), the Vinnova NFFP6 Project 2013-01206, the Swedish Foundation for Strategic Research (CUAS Project), the Swedish Research Council (VR) Linnaeus Center CADICS, and the ELLIIT network organization for Information and Communication Technology. We thank Alexander Kleiner for initial discussions concerning the topics in this paper.

\section{References}

[1] Jose Joaquin Acevedo, Begoña C Arrue, Ivan Maza, and Anibal Ollero. Cooperative large area surveillance with a team of aerial mobile robots for long endurance missions. Journal of Intelligent $\&$ Robotic Systems, 70(1-4):329-345, 2013.

[2] N. Agmon, S. Kraus, and G.A. Kaminka. Multi-robot perimeter patrol in adversarial settings. In IEEE International Conference on Robotics and Automation (ICRA), pages 2339-2345. IEEE, 2008.

[3] Noa Agmon, Gal A Kaminka, and Sarit Kraus. Multi-robot adversarial patrolling: facing a full-knowledge opponent. Journal of Artificial Intelligence Research, 42:887916, 2011.

[4] J.W Barnes, V.D Wiley, J.T Moore, and D.M Ryer. Solving the aerial fleet refueling problem using group theoretic tabu search. Mathematical and Computer Modelling, 39(6-8):617 - 640, 2004. Defense transportation: Algorithms, models, and applications for the 21st century.

[5] F. Bullo, J. Cortés, and S. Martínez. Distributed Control of Robotic Networks. Applied Mathematics Series. Princeton University Press, 2009.

[6] H. Choset. Coverage for robotics - A survey of recent results. Annals of Mathematics and Artificial Intelligence, 31(1-4):113-126, 2001.

[7] T. H. Cormen, C. E. Leiserson, R. L. Rivest, and C. Stein. Introduction to algorithms. MIT press, Cambridge, Massachusetts, London, England, 3rd edition, 2009. 
[8] Jason Derenick, Nathan Michael, and V. Kumar. Energy-aware coverage control with docking for robot teams. In Intelligent Robots and Systems (IROS), 2011 IEEE/RSJ International Conference on, pages 3667-3672, Sept 2011.

[9] R Huisman. Scheduling the refuelling activities of multiple heterogeneous autonomous mobile robots. Master's thesis, Delft University of Technology, 2014.

[10] Zhipu Jin, T. Shima, and C.J. Schumacher. Optimal scheduling for refueling multiple autonomous aerial vehicles. Robotics, IEEE Transactions on, 22(4):682-693, Aug 2006.

[11] Jonghoe Kim, ByungDuk Song, and James R. Morrison. On the scheduling of systems of UAVs and fuel service stations for long-term mission fulfillment. Journal of Intelligent \& Robotic Systems, 70(1-4):347-359, 2013.

[12] S. Lazebnik. Visibility-based pursuit-evasion in three-dimensional environments. Technical report, University of Illinois at Urbana-Champaign, 2001.

[13] Y. Litus, P. Zebrowski, and R.T. Vaughan. A distributed heuristic for energy-efficient multirobot multiplace rendezvous. Robotics, IEEE Transactions on, 25(1):130-135, Feb 2009.

[14] Neil Mathew, Stephen L Smith, and Steven L Waslander. Multirobot rendezvous planning for recharging in persistent tasks. Robotics, IEEE Transactions on, 31(1):128-142, 2015.

[15] Anton Molyboha and Michael Zabarankin. Stochastic optimization of sensor placement for diver detection. Operations Research, 60(2):292-312, 2012.

[16] T. Shermer. Recent results in art galleries. Proceedings of the IEEE, 80(9):1384-1399, 1992.

[17] I. Suzuki and M. Yamashita. Searching for a mobile intruder in a polygonal region. SIAM Journal on Computing, 21(5):863-888, 1992.

[18] Koji A. O. Suzuki, Paulo Kemper Filho, and James R. Morrison. Automatic battery replacement system for UAVs: Analysis and design. Journal of Intelligent $\&$ Robotic Systems, 65(1-4):563-586, 2012. 